\title{
THE CONCEPT OF FORMATION OF ECONOMICALLY SAFE LIFE CYCLE OF AGRICULTURAL ENTERPRISES
}

\author{
Agibalov A.V., Candidate of Economic Sciences, Associate Professor \\ Zaporozhtseva L.A., Doctor of Economic Sciences, Professor \\ Tkacheva J.V., Candidate of Economic Sciences, Associate Professor \\ Voronezh State Agrarian University named after Emperor Peter the Great, \\ Voronezh, Russia \\ E-mail: agi-64@mail.ru, ludan23@yandex.ru, julchen19@yandex.ru
}

\begin{abstract}
In the modern economy the securing of economically safe development is a daily task for top management. It allows not only to recoup the investment in the commercial structure and to avoid global financial crises, but to prolong the period of stability of the organization. The particular importance of this task becomes in the conceptual plane of agribusiness development and agriculture in particular. Financial support planned for implementation in the industry, has no analogues in the history of the Russian economy of the last decades, and therefore requires tools to ensure its long-term effect. It is impossible to implement it without the conceptual transformation of basic theory of operation of enterprises, which explain the regularities of their development from inception to closure. The present paper suggests that the basis of economic security of activity is guarantee stability of growth at each stage of the organization development. Drawing on extensive literature review, is defined possibility of replacement of an equifinality reference point of the enterprise development to permanent on the research basis of the various continuance business cycles. It allows providing for stability of the business units development in long-range outlook.
\end{abstract}

\section{KEY WORDS}

Lifecycle, economic security, threats, opportunities, stage, an equifinality reference, strategy, agribusiness.

The theory of lifecycles allows not only tracing regularities of the economy agents development and their organizational structures at different stages, but also to operate their movement direction. In turn lifecycle curves are able to give the system conception of the organizational problems and the relations appearing in activity of economic formations of agribusiness. Thereupon the task of the theories adaptation of lifecycle is acquired actuality to the solution of the problem of guaranteeing economic security development of the commercial organizations in the long-term outlook.

\section{ECONOMIC SECURE LIFECYCLE OF ENTERPRISES}

As development every economic and social system functions not chaotic. There is a fixed regularity by passing of number stages which change has round-robin character by it. Cycling means existence of some phenomena sequence regularity and processes during any period which is called as a cycle. In the context of the commercial organizations development are formed the economic cycles periodic and, as a rule, irregular character and which are defined by fluctuations round a long-term trend. They represent the repeating fluctuations of economic activity (an economic conjuncture), pressing (economic slack, recession, depression) and economic expansion (economical upturn). At that determinists consider that their reasons are predictable, positive factors which are formed at the raising stage (recession factors) and recession (raising factors). Therefore, any movement can be operated and has a predictable trajectory. The stochastic point of view based on position that cycles are generated by factors of the accidental nature and is a reaction of economic system to internal and external impulses. Thus is made up a conclusion that the theory of 
chaos exists. But, it is proved, taking into account existence of interaction between order and chaos in the arising systems that even in chaotic processes there are definite schemes of development [10].

Both groups of scientists recognize existence macro - micro and meso - cycles. To micro business cycles are related stages of the organizations development, and mesobusiness cycles include the stages of products lifecycle, of the markets lifecycle, of the company employees (showing stages in evolution of personal career of a worker) and others.

Depending on duration are differentiated four types of macroeconomic cycles: the short-term - Kitchin's cycles connected with fluctuations of world reserves of gold continuing 3-4 years [11]; the medium-term - Juglar 's cycles formed in the sphere of the money and credit in circulation which last 7-11 years [9], the long-term - the Kuznets cycles called construction, frequency of 15-25 years [13] and big business cycles of Kondratyev frequency of $45-60$ years [12]. The nature of cycles in modern economy is studied by the analysis of external and internal factors.

Short-term business cycles [13] (with the distinctive period of 3-4 years) were opened in the 1920th years by the English economist J. Kitchin. The reason of short-term cycles the scientific explained as the fluctuations of world reserves of gold. Thus today such explanation can't be considered reliable and satisfactory. The mechanism of these cycles generation in the economic theory is connected usually with the temporary logs in the movement of information influencing decision-making by firms [2]. The firms react to improvement of an environment with a full load of power, the market is filled with goods, and further the warehouses are full of excessive stocks of finished commodity. Then it is decided to decrease the load of power, but with a certain delay. Because the information of excess of supply over demand is formed with a certain delay. As economic subjects have to control and estimate this incoming information, they aren't able to adapt timely for fluctuations of the environment. And it is required certain time for renewal of their normal operation.

Juglar's cycles are called by name of French economist K. Juglar. He was one of the first who described these cycles. They trace also fluctuations of investment volume into basic stock. Medium-term business cycles (with the characteristic period in 7-11 years) are characteristic fluctuations not simply in the level of power loading and volume of commodity stocks, but also fluctuations in the investment volume into basic stock. As a result to the temporary delays inherent in Kitchin's cycles add the temporal log. This temporal log takes place between the adoption of investment decisions and the erection of the suitable power, and also between the erection and the relevant starting. The additional delay is formed between the recession of demand and the liquidation of the industrial power. It conditions their longer period, in comparison with Kitchin's cycles. Negative cyclic economic crises (recessions) can be considered as one of phases of the cycle of Juglar together with recovery phases, expansion phases and a depression. In general view at 7-11 a summer cycle of Juglar are allocated the following four phases with specification for subphases:

- the revival phase with the subphases of start and acceleration; boom;

- the phase of raise (prosperity) including a subgrowth phase and an overheat, or

- the recession phase which has the subphases of failure and/or an acute crisis and recession;

- the phase of depression (stagnation) with the subphases of equalization and shift

Long-term business cycles which last about 15-25 years are called as cycles (rhythms) of Kuznets. They get the name of American economist, future Nobel Prize laureate Simon Kuznets who proved their existence in 1930. Kuznets connected the reasons of appearance of long-term waves with demographic processes, such as inflow of immigrants and construction changes. Therefore he called them "demographic» or "construction» cycles. Besides the Kuznets rhythms are considered in system of the technological and infrastructural cycles meaning the mass renovation of the main technologies. It should be noted that it isn't observed the accurate frequency in their changes, in spite of indisputable being of such cycles. Therefore research workers register mean value in 15-25 years.

Depth of these crises (short, medium and long-term) can be aggravated depending on a phase of a big wave. 
Cycles (waves) of Kondratiev are periodic cycles of changes in modern global economy. They prolong 45-60 years. Kondratiev explained regularity of the character of longterm fluctuations by inequality of scientific-and-technological advance together with the length of the capital turnover, and also a variety of reasons of social and historical character [12]. Kondratiev revealed the long-term mechanism making for periodic structural transformation of economic system: the renovation of technological base and productive facilities, the reorganization of the economic mechanism and organizational structure. He expressed the opinion about possibility of the social and economic system creation. It would be generating the long fluctuations, providing the predicted movement from one steady state of the economy to another.

Connection between long cycles of Kondratiev and medium-term cycles of Juglar is confirmed by correctness of alternation of buoyant and bearish phases of Kondratiev waves. Each phase of 20-30 years is defined by the character group of nearby medium-term cycles.

As commercial entities are part of macroeconomic, then with macroeconomic cycles are developed theories of cycling at the level of the organizations. They had a wide distribution and development in the middle of the 20th century.

The unanimous opinion of scientists about industry cycles, as independent factors of crisis development of enterprises does not exist. However, there is evidence of long-term studies (1972 - 2011) in forecasts of complex natural conditions of the harvest by I. Sagitov, indicating the presence of biological cycles in crop production. The assessment of conditions on the technology "ZONT» allows with quality lag to calculate the forecast impact biological resources one of the basic sub-sectors of agriculture and, consequently, to justify the amount of recoverable costs. The last factor is decisive in ensuring the effective functioning of agricultural and all of AIC [18].

At the micro level cycling is lifecycle of activity of economic entities. In economic literature there are various models of their life cycle. But it doesn't allow to build simple process of adoption of administrative decisions and to provide economically safe development in the course of the period of their functioning.

The theory of lifecycles of the organization is one of the widespread modern evolutionary and teleological, natural models considering organizational development as a certain analog of developments of a biological organism. The theory allows establishing; at what stage of lifecycle is the enterprise, to prognoses the direction of its development and rise of critical situations. It gives the chance to be prepared for them properly that is provided with receipt of aprioristic information and concentration on the solution of real problems.

Development of the organization by scientists is understood as natural, stage-by-stage and in many respects the programmed process providing inevitable passing of a number of consecutive stages by it thus opportunity to pass any of them is excluded. Step by step are changed logic of functioning of business and type of strategy, is decreased flexibility of response to external changes, but thus is increased ability to control in the organization.

The theory of lifecycles of the enterprise is development and a component of natural model of the theory of the organization and management. Primarily the model of lifecycle was developed on the basis of an assessment of the government organizations activity, and then was created model for the commercial and noncommercial enterprises. The concepts of lifecycle studied changes of various parties of business structures during the period of its functioning: technological changes, internal culture and efficiency, the varying priorities of top managers. It were researched lifecycle of again created organizations, the industrial enterprises, universities, hospitals, publishing houses, service companies and others.

Research of regularity and cycling of development of the organizations was carried out by the famous expert in the field of the general theory of systems K. Boulding [3]. He was one of the first. He proved that for each of phases is characteristic the features of functioning - from the birth till a maturity, finishing with death.

D. Miller and P. Frizen described the stages of lifecycle as a configuration of certain contextual and structural variables [14]. Somewhat different version about the theory of the organization lifecycles was described by A. Chandler- the follower of empirical approach. At this point was connected the changes of the organization activity with the changes of its relationship with environment. At this point was connected the changes of the organization 
activity with the changes of its relationship with environment. In his opinion, the organization develops types of structures in response to the general growth and invitation of the market [4].

S.G. Hanks thinks that the model of lifecycle «... can provide the card of a way identifying critical organizational transitions, and also traps which the organization has to seek to avoid during the growth in sizes and complexity. The exact model of lifecycle can provide the schedule of addition of management levels, formalizing organizational procedures and systems and revising priorities of the organization. It can help know management when to refuse the strategy or procedures which are carefully grown up in the past, which only hinder from the future growth» [7].

One of the most significant concepts of the enterprise lifecycle is the models developed by L. Greyner and I. Adizes. In their opinion functioning of the organization represents the process of change of one stage by another. But each of stages is characterized by a certain feature set.

Of L. Greyner's opinion the major criteria of lifecycle model in the organization are: age and size of the organization, branch rate of increase, stages of evolution and stages of revolution [6]. From his point of view, there are stages ("growth stage») in development of the enterprise which change is caused by approach of crisis at the previous stage. Each stage of development is the reason following and result of the previous.

Stages of lifecycle of the economic agent are more fully presented in I.K. Adizes's theory. In contrast to other models, it is complete and includes the most detailed list of stages from «birth» to "death». Here is specified the zone of the maximum development of the enterprise - a stage of «blossoming» [1].

The Russian researchers consider a lifecourse of the organization in business from the sociocultural point of view. They base on personal experience of management consultation. They define four stages of lifecycle of organizational development, different from offered by other researchers [5]. For each of certain stages they give the characteristic of the enterprise organizational structure, system of relationship between employees and environment:

The first stage - a Thrash - is characterized with predominance of the informal relations, «communal» mentality, equality, ideological (charismatic) leadership and nonprofessional (friendly or paternalistic) management.

The second stage - Mechanization - is defined by universal formalization of the relations, processes and procedures. The enterprise passes to regular management, division of labor is extended, are formed the official regulations and the rules of internal order, is formed the process of standardization and utility.

The third stage - Internal Business - as the main task of the business organization is increase of efficiency of activity. This stage differs from others in delegation of powers, decentralization of the power, internal enterprise initiatives of employees and divisions which are regulated by policy and of the organization strategy;

And the last, fourth stage - Quality Control - is characterized by the fact that the company sights at capture of strategic priorities in the market thanks to creation of own quality standards.

Each of these macrostages is subdivided into six stages: formation, rise, stabilization, stagnation, crisis, disintegration.

From this it follows that the scientists consider existence of the commercial organizations lifecycles. But they have not unanimous opinion about quantity of their stages, their names and sequence of removability. We established also there is an united opinion that the sequence of stages of lifecycle in the enterprise is in existence, in which each stage is a sequent of the previous. Any phase has special characteristics, but development doesn't carry strict determination.

In that way it is necessary to understand, what are the basic factors defining a business development stage and how they are changed in time?

There are marked out the following factors forming the stages of the enterprise lifecycle: the organizational set-up, the production systems, the information processing procedure, the strategy and the environment [7]. Their combinations bring to creation of a small number of the general configurations repelling the general organizational structures 
and the creation scenarios of the strategy, the advancing results or the intermediate results and another. Therefore, it is possible to describe the stage of the organization lifecycles as "a unique configuration of the variables connected with an organizational context and the structure».

The mainframe factor of change of the favorable stage of the organization lifecycle on the negative stage is the conflict in the organizations. So mean the scientists. The conflict is often connected with unwilling of management to include an establishment point of feedback with the organization employees in the list of the company purposes. As a result the quality of decision-making is suffered and there comes the period of recession [15].

It is also possible to use the theory of production factor with a view to do the detailed analysis of the enterprise development for lifecycle stages [8]. Each of these six factors characterizes a certain aspect of the enterprise development:

- a human factor - people development;

-a technical and technological factor - the enterprise technological development and development of the technologies applied by it;

-a material factor - increase in economic indicators of the company activity;

-an institutional factor - development of institutes, introduction of professional management system (management);

-an organizational factor - development of the enterprise organizational set-up;

-an informative factor - improvement of the information streams use for precipitation of the organization development and increase of the informative openness.

Analysis of the development dynamics of these factors allows tracing more precisely the enterprise development over the time of its existence. Use of O. V. Inshakov's theory will allow to foretell in advance the forthcoming problems in the company and to find more favorable ways of their decision. Harmonious combination of these factors, their system unity is capable to maximize the enterprise efficiency, provided achievement of the development benchmark and the timely lift on stages of lifecycle. Unsafely and imbalance of the factors of production combinations can be considered as a danger to the normal enterprise development.

We consider in detail the influence of each of the earlier enumerated factors at different lifecycle stages of the enterprise. We use the theory of lifecycle of I.K. Adizes as the most proper model describing the organization development during its existence. This method includes ten stages of the enterprise lifecycle: Courtship, Infancy, Go - go, Adolescence, Prime, Stable, Aristocracy, Salem City, Bureaucracy, Death, the figure 1. [1].

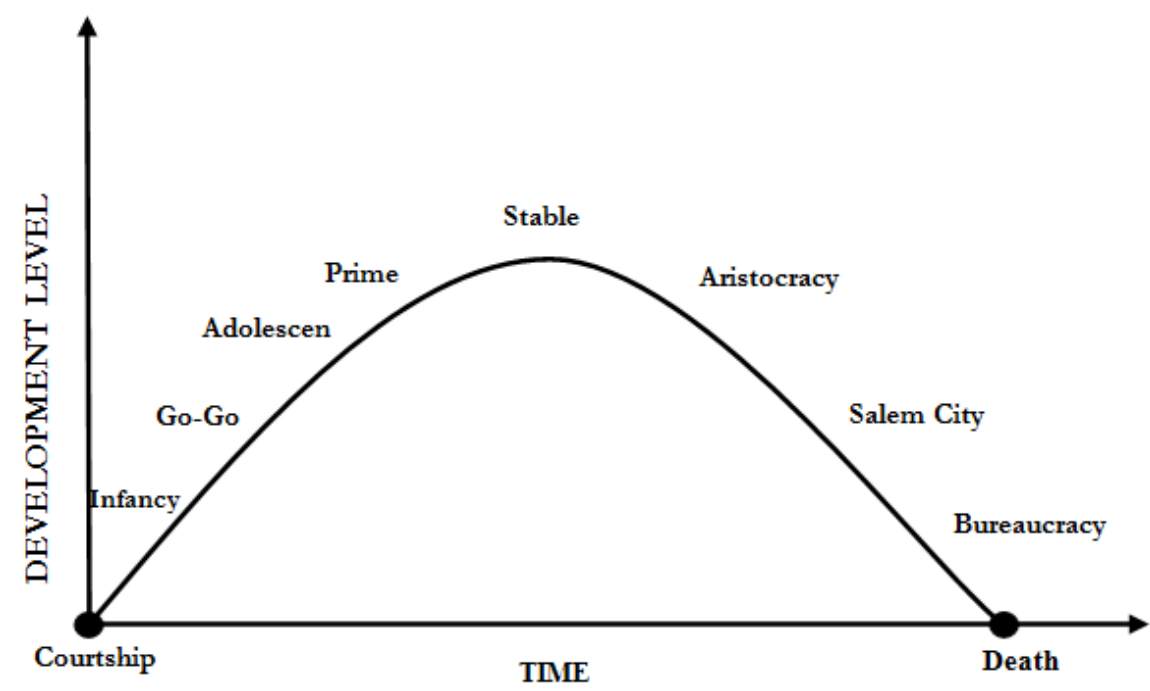

Figure 1 - The enterprise lifecycle [1]

We consider development and influence of factor of production on each of the presented stages of the enterprise lifecycle. The total conformity combinations of factors of 
production to the offered characteristics of each stage are a sign of the organization readiness for transfer to the following lifecycle stage. At the stage "Courtship» the enterprise wasn't come into the world yet and exists only as an idea.

The birth of the organization happens when the founder assumes risk, forms a package of documents and realizes even one «trial» production cycle. The fundamental factors defining this stage of the enterprise lifecycle are human and informative. Other factors influence will be minimal as juridical the organization doesn't exist yet and consequently production factors aren't involved.

The stage of "Infancy» is defined by the beginning of the economic structure activity without the exact created policy and the budget. Degree of centralization of its activity is enough. It functions uncoordinated by weak service hierarchy and therefore collides with high probability of the fast problems escalation into crisis situations. At this stage the main factor for a survival and the organization development there is a level of interest and qualification of the founder- leader (as a rule in one person or the leader - one of founders). There are the missing factors of production: the organizational structure, the regulations of the work organization, the capital stock, the cash flow, the inflow of the employees. Influence of all six factors is different, and the level of their development has possibilities for rise.

The stage "Go-Go» is described by the fast rates rise of trade, expansion of supplier base and contract acceptors, of the company sizes increase. It is positively repelled in financial indicators. The founder is irreplaceable. It is attached little importance to organizational structure, management processes, trust and system of remuneration. The property owner is interested more in joint enterprises, in strategic corporations. The activity organization is characterized by the vague communications, fuzzy trust, and internal disorganization. At that the enterprise activity is flattend out and the formal structure is created. Development degree of four of six factors is estimated as high. But the influence increase of two others in part of institutionalization of the enterprise activity and increase of the corresponding competences at employees is necessary. The stage "Adolescence» is defined as a rebirth and the enterprise "growing", by transition from individual management of the company owner to professional management.

The stage "Adolescence» is defined as a rebirth and the enterprise "growing», by transition from individual management of the company owner to professional management. Development degree and influence of human, technical and technological, institutional and information factors are quite high. But for all that influence of material and organizational factors is decreased, owing to a quality priority over quantity.

The stage "Prime» is described achievement of the enterprise optimal condition, achievement of balance between self-checking and flexibility. The loftiest development of all factors of production leads to the justified leadership of the enterprise in the market. Management process is completely institutionalized: professional management supports close connections with the contractors that allow to create an optimum product and to get the maximum profits. The enterprise disposes of functional systems and organizational structures, the development level and influences of all factors is maximum. The organization takes the steadiest position.

The stage "Stable» or "Ageing» is called ambiguously as a result of the translation and specific of the economic substance. As this stage is characterized on the one hand by peak of the maximum factors influence defining lifecycle of the enterprise development named the top of «iceberg», on the other hand it is a critical point between rise and its loss. In other words since this stage the curvilinear motion of lifecycle represents a come-down process. All external characteristics of the achieved success during "prime» are remained, thus the enterprising spirit disappears and grows conservatism degree. Relying on the cumulative competitive privileges, the development level of other factors of production at this stage is remained quite high.

The stage "Aristocracy» is inherent in the senescent organization which isn't focused on the strategic objectives achievement. The enterprise is contented with last achievements, without planning future rise. Financial resources are used on appropriation of luxuries or satisfaction of identified needs of founders, dividends are paid on aggressive type, 
modernization of the equipment isn't made. It leads to the ineffective spending of resources and decrease of the material factor in the level of development. Other factors are invariable.

The stage "Early Bureaucracy» or "Salem City» is marked by appearance of the interpersonal conflicts at the enterprise; search the guilty of the current situation, degradation of the employees' qualification that leads to decrease of the human factor development in the level. The number of branches and divisions of the company is reduced, the level of the organizational factor development is reduced too, but its negative influence is raised. The level of the information factor development is low too. There is no strategic vision and planning. Decrease in the development level of all six factors of production to low values, together with their negative dynamics; conduct the enterprise to decrease in activity efficiency and impossibility of normal functioning without any cardinal changes.

The stage "Bureaucracy» is defined as the temporary organization liveness at the expense of external subsidies. The production is closed; the development level of technical technological factor is extremely low. The enterprise is finally isolated from environment, being focused in itself. It is the enterprise overstocking of finished goods or refusal of consumers of company services, the difficult financial position leads to release of employees. As a result the enterprise turns into the finished bureaucratic structure concentrated only on rules and norms.

The stage «Death». The company ceases to exist in view of impossibility to bring the useful production to society or to provide its solvency. The enterprise goes bankrupt and stops the existence. Development levels of all factors of production decrease to zero.

It is expedient to consider development of six factors of production in stages of the enterprise lifecycle, considering as described. The analysis of its dynamics allows tracing more fully the organization development during its existence period. And it is therefore better to predict the forthcoming problems and to find more profitable solutions. It is undoubtedly, will affect the management quality. With high certainty it is possible to claim that the highest extent of economic perils appears in the "Prime point» - at the time when the enterprise reaches the sacred stability and doesn't know where to move on. So, achievement of the stage "Prime» by the organization means the maximum development of all six factors of production. Consequently, the main reason of the problems appearance in the company is its defective development typical for certain stages of lifecycle. Besides, the organizations are capable not only to remain long time at one stage of development, but also very quickly move to decline and death.

Using cyclic method, it is possible to suppose that danger of economic security are formed at the turn of long, medium and short waves of economic development and realized in the transition form to a new cycle of economic development, or as a result of loss of competitive edges.

In our opinion, the main objective of providing economic security of the enterprises is not achievement of the highest degree of its stability, but providing increase and development now. Of what source of financing the commercial organization reaches this goal, doesn't matter: there are enterprises capitally stable, but stagnating, and, vice versa, unstable, but rash developing. As is easy to see the last are the most successful. It is related to the fact that all changes happen in the solution process of any problems. The scientists note the changes and problems are linked, they always arise together and the development success of the company depends on timeliness and efficiency of the arising problems solution [17].

From our point of view the economic security strategy of the enterprise has to be based on creation of such business environment of the enterprise which will promote maximizing profit. And at the same time will provide both its rise and dividend payment to owners.

The concept of compromise between risk and earning power consists in the proceed obtaining in business. It is often agreed to risk, and the connection between these two interdependent characteristics directly-proportional: the higher expected or required return (in other words return of capital employed) the higher risks profile connected with possible non delivery of this return. 
Consequently, in our opinion, providing economic security of the commercial organizations has to include the control of danger of its loss and providing of the enterprise sustainable development.

At this point of research there is a need of elaboration of such concepts as "safety", «stability», «development», and also an establishment of interrelations and contradictions between them.

"Development» and "stability» are basic under the understanding of the economic security entity and formation of accesses to its providing [16]. "Development» is one of the components of the economic security. Thus not any development answers purpose of safety. Development has to be followed by environmental compliance, social norms. Besides, high rise rates have to be reached by providing with significant prosperity marks for the public. If the organization doesn't develop, it is sharply reduced possibility of survival, and also strength and flexibility to internal and external dangers.

"Stability» and "safety» are the most important characteristics of the enterprise economy as unified system and its state. "Stability" of the enterprise economy repels integrity of its elements, vertical, horizontal and other connections in system. As well as ability to observe internal and external forces, to restore the established normal state after its sudden violation by any external or internal factor.

From the point of view of natural sciences, "sustainable development» is a complete historical process of development security. As if the organization doesn't care of the reached development security, it is doomed to decline. Not only now, but also in the future development preservation is interpreted as «preservation of not decreasing rise rate of efficiency of the full power use at all times or not decreasing rise rate of available capacity» [16].

In turn «safety» is the object condition from the point of view of ability to survival and development in the neutralization conditions of internal and external dangers, and also actions of the unpredictable and difficult predicted factors.

The steadier is the enterprise the more viable it is, at that the assessment of its safety will be sufficient high. But further, as a rule, it is necessary to expect the proportions violation and the connections between different components of system. It conducts to its destabilization and is a signal of the enterprise transition from the secure to a dangerous state. As a result, the dynamics of development is steadier; the probability of danger is less.

Consequently it is possible to claim that stability can't be out of development. Any developing system periodically transits from one steady state to another, that is has a certain the development regularity.

The problem of preservation of such development consists in denial of unstable states at the enterprise and the approaches elaboration of cyclic transition from one steady state to another on a trajectory of a sustainable development, liquidating dangers to security of economic entities. It is possible to allocate at least three functional criteria generating a financial component of the enterprises economic security: profit return, revenue and owned capital. In the trine the main driving force and criterion of dangers control function and the enterprise ensuring development, in our opinion, is the profit return. Rise of revenue provides the enterprise development, and increase in owned capital is its financial rise and stability of the capital. It is necessary to recognize an economic added value of the enterprise as the indicator making a synthesis of these characteristics.

So, it is possible to formulate the following determination of stability as the enterprise abilities long-term to resist to the destructive fluctuations. The destructive fluctuations are due to the fact of environment changes and the internal reasons. The enterprise thereat must effectively discharge operations peculiar to it. In this definition the attention is focused on the long-term principle of the enterprise opposition to destructive fluctuations as a result of complexity of providing all components of stability. It promotes preservation of optimal parameters of system development.

Timely tracking of opportunity and the reasons of kind change of financial stability towards phases of its providing (absolute and normal) or phases of its loss (unstable and crisis) will allow to manipulate, from our point of view, the size and structure of property, 
financial resources and competitive advantages of the enterprise for the purpose of providing its sustainable development.

It is necessary to trace economic security on all stages - throughout all life of the enterprise for ensuring its strategic (long-term) safety. Thus the logic of ensuring strategic safety has to be brought to an assessment of dangers and their immediate elimination. It is possible to identify stages of lifecycle most precisely: birth, rise, maturity, recession and crisis. It is important to establish, in our opinion, achievement of maturity, as highest stability degree is the most dangerous condition of the enterprise.

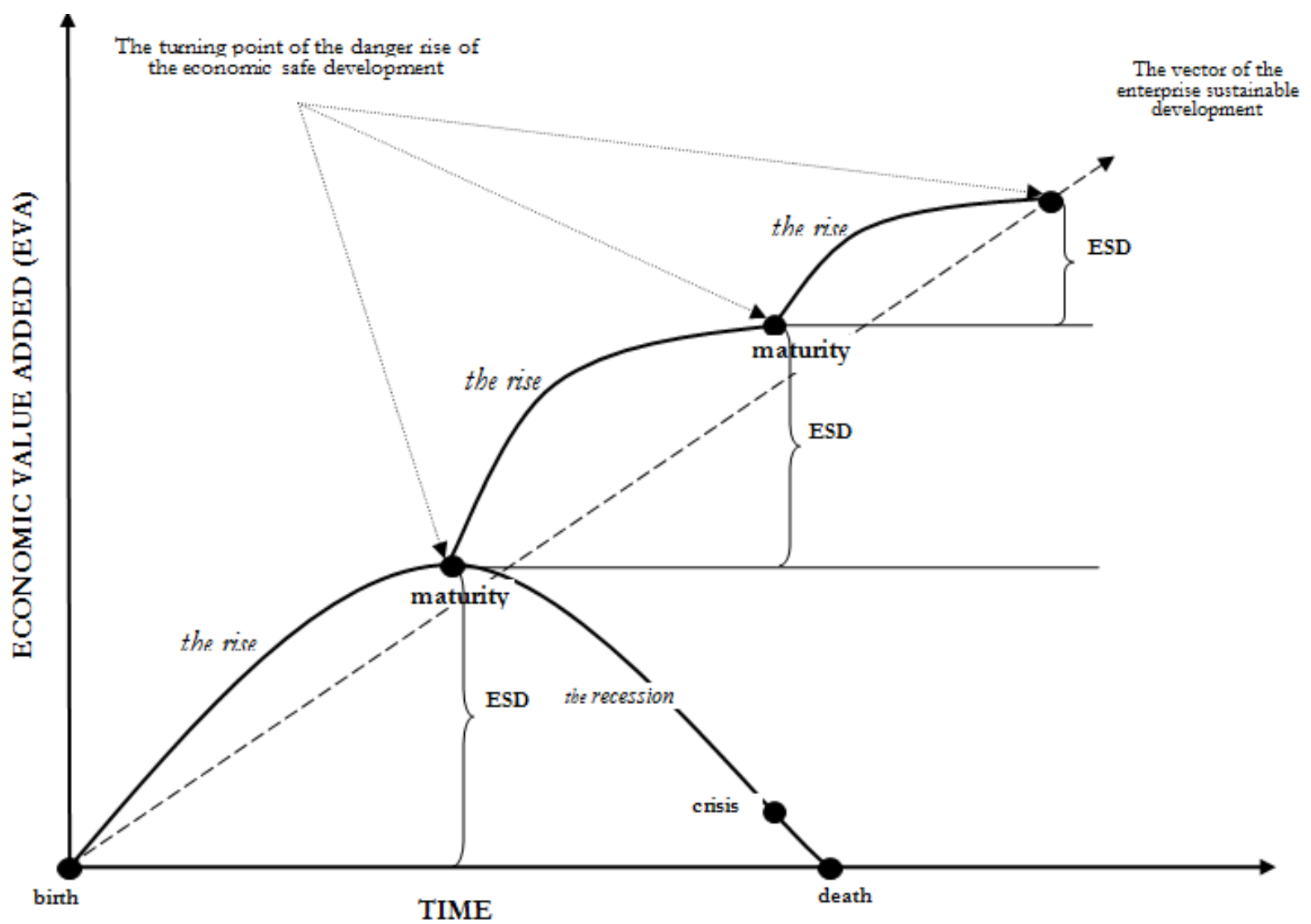

ESD - economic safe development

Figure 2 - Regularities of cyclic and economically safe development of the enterprise in the current context

With the object of the living trajectory of the commercial organizations was strategically safe, it is necessary, to find the ways for the new rise, locating in the point of maturity (fig. 2). Transformation of the economic security danger in possibility of the organization development assumes the fastest leveling of recession problems - solution of the internal conflicts and the choice of perspective points of rise including the basis of an assessment of biological factors that are particularly important in agriculture. Thus, a priori prediction of yield allows to compare the investment of resources in production, costs per hectare of sown areas of arable land and to return them in absolute and relative amount of financial result.

Consequently the economic security conception of the enterprise allows proving the opportunity and the replacement conditions of the equifinality reference point of the enterprise development on the permanent. It is reached by maintenance of the constant, continuously proceeding development of the enterprise, replacing the maturity stage with new rise, without having allowed recession and crisis. Possibility of securing such development is connected with necessity of theoretically-methodological development and invagination of the presented providing strategy of economic security in activity of the commercial organizations in the long-term prospects [19]. 


\section{CONCLUSION}

Thus the basis of economically safe development of enterprises is a stage-by-stage sustainability. Replacement of the equifinality reference point of the enterprise development on the permanent explains the mechanism of maintaining this sustainability. So, the danger of a movement towards decay and death is conceptually transformed into the possibility of cyclic transition to a higher trajectory of sustainable development based on the strategic economic security in the context of the enterprise lifecycle. It allows to identify dangers in a determinant section of the enterprise safety at the micro level (business processes and resources), to offer highlight figure of safety and their corridor parameters, and on this basis to create the functioning mechanism of providing strategic economic security of the enterprise. The provision of economically safe development of agricultural enterprises in the framework of the concept of the life cycle in turn is the key to long-term strategic investment efficiency and support of the food subcomplex AIC.

\section{REFERENCES}

1. Adizes I.K. Managing Corporate Lifecycles. United States, 2004. 484 p.

2. Aukutsionek S.P. Modern Bourgeois Theories and Models of the Cycle: a Critical Analysis. M.: Nauka, 1984. 223 p.

3. Boulding K. The Economics of Knowledge and the Knowledge of Economics. American Economic Review, 1966. 56. (1), p. 1-13.

4. Chandler A.D. Strategy and Structure: A Chapter in the History of Industrial Enterprises. Cambridge, Mass, MIT Press, 1962. 210 p.

5. Emelyanov E.N., Povarnitsyna S.E. The Psychology of Business. Moscow, 1998. 511 p.

6. Greiner L.E. Evolution and Revolution in the Growth of Organizations. Vestn. St. Petersburg Univ. Ser. Management, 4. 2002. p. 76-94.

7. Hanks S.H. The Organization Life Cycle: Integrating 8. Content and Process. Journal of Small Business Strategy, 1990. 1, p. 1-13.

8. Inshakov O.V. Theory of Production Factors in the Context of Development Economics: the Scientific Report at Presidium IAOS (Moscow, 29 Nov. 2002). Volgograd: VoISU Publishing, 2002. p. 89

9. Juglar C. Des Crises Commercials Et De Leur Retour Periodique En France. Paris. 1862.

10. Kauffman S. The Origins of Order: Self Organization and Selection in Evolution. New York: Oxford University Press, 1993. 709 p.

11. Kitchin J. Cycles and Trends in Economic Factors. Review of Economics and Statistics, 1923. 5 (1), 10-16

12. Kondratiev N. Large Cycles of Conjuncture and Theory of Foresight. Selected Works. M.: Economics, 2002. 768 p.

13. Kuznets S. Secular Movements in Production and Prices. Their Nature and their Bearing upon Cyclical Fluctuations. Boston: Houghton Mifflin3. 1930.

14. Miller D., Frizen P. Longitudinal Study of the Corporate Life Cycle. Management Science, 1984. 30(10), p. 1161-1183.

15. Morrill C. The Executive Way: Conflict Management in Corporations. Chicago: Univercity of Chicago Press, 1995. 328 p.

16. Senchagov V.K. Economic Security of Russia: General Course: Textbook. M.: Delo, 2005. $896 \mathrm{p}$.

17. Watzlawick P., Weakland J., Fish R. Change Principles of Problem Formation and Problem Resolution. New York: Norton Books, 1974. 172 p.

18. Zagaitov I.B. The objective laws of fluctuations of weather conditions and technology «ZONT». In the collection of the Russian agro-industrial complex development potential Collection of scientific papers on the results of inter-regional scientific-practical conference. 2013. p. 259-268.

19. Zaporozhtceva L.A. The Life Cycle of the Company and its Strategic Relationship with the Level of Economic Security. Social-Economic Phenomena and Processes, 2014. 9 (12), p. 81-89. 